OR THE DESTABILIZING EFFECT OF DAMPING

IN NONOONSERVATIVE D.ASTIC SYSTEMS

by

G. Herrmann

Professor of Civil Engineering

The Technological Institute

Northwestern University

Dranston, Illinole

and

Inf.-Chang Jong

Graduate Student, Dejartment of Civil Engineering

The Technological Institute Northwestern University

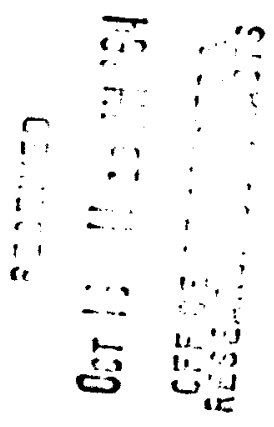

Evanston, Illinois

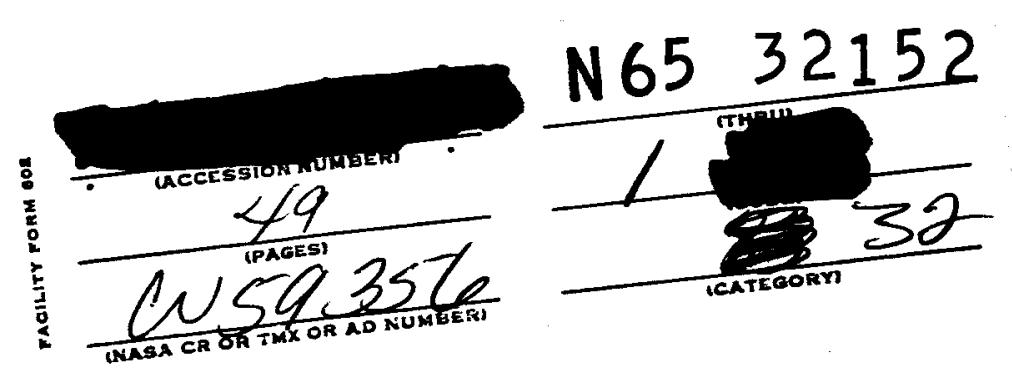

1 This research was supported oy the National Aeronautics and Space Adinistration under Grant NsG 605.

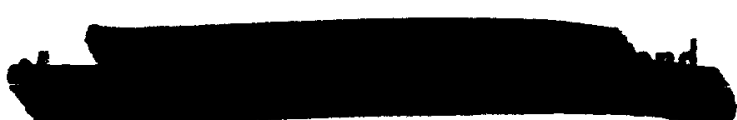


The destabilizing effect of Inear viscous damping in a nonconservative elastic system is investigated by studying the roots of the characteristic equation in addition to the stability criteria and by introducing the concept of degree of instability. A generic relationship between critical loadings for no damping and for slight damping as well as vanishing damping is established. It is found that while the presence of small damping may have a destabilizing effect, proper interpretation of the limiting process of ranishing damping leads to the same critical load as for no damping.

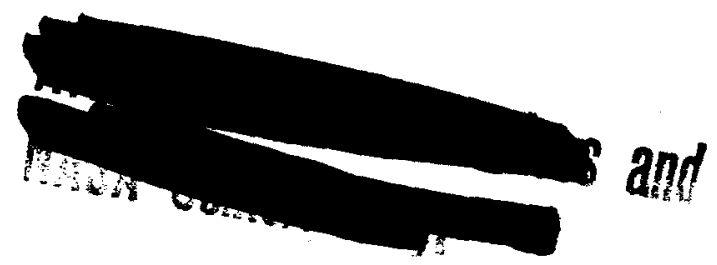


Introduction

It has been discovered by ziegler [I] a little more than a decade argo, that internal damping may have a destabillzing effect in nonconservative elastic system. He considered a double pendulum with viscoelastic hinges as a model of an elastic bar with internal damping and let a tangential force act at the free ond. The critical loading obtained in complete absence of damping was found to be considerably higher than by including damping at the outset of the analysis and then letting the damping coefficients approach zero (vanishing damping) in the expression for the critical force.

This rather surprising and paradoxdcal finding was ascribed in later studies by Ziegler [2], [3] to the possibility that internal damping is inadequately represented by linear damping forces which are linear combinations of the generalized velocities and that the hysteresis effect should be taken into account.

The destabilizing effect of damping was further elaborated upon by Bolotin [4] who considered a general two-degree-of-freedom system not related to any particular mechanical model and who found additionally that the destabilizing ef fect in the presence of slight and vanishing damping is highly dependent on the relative magnitude of damping coefficients in the two degrees freedom.

It is the aim of the present investigation to make an attempt at supplying some additional insight into the destabilizing effects of Inear velocity-dependent damping in nonconservative systems, without raiging the question here as to the suttability of this demping nechantst 
for a realistic system. For this purpose the system discussed by Ziegler is reconsidered, and not only the stability conditions are investigated but also the roots of the characteristic equations themselves. Plots of these roots for various ranges of loading illustrate graphically how the paradoxical effects of vanishing damping are generated. Further, the results of the mathematical stability investigations are interpreted in physical terms by introducing the concept of degree of instability.

These concepts permit to carry out a gradual transition from the case of small damping to the case of vanishing damping and relate them to the case of no damping. Finally, some remarks are made with regard to possible behavior of an elastic bar with distributed parameters.

The Model

We consider a double pendulum, Fig. 1, composed of two rigid weightless bars of equal length $l$, which carry concentrated masses $m_{1}=2 \mathrm{~m}$, $m_{2}=m$. The generalized coordinates $\varphi_{1}, \varphi_{2}$ are taken to be small. A load $P$ applied at the free end is assured to be acting at an angle $\varphi_{2}$ (follower force). At the hinges the restoring moments $c \varphi_{1}+b_{1} \dot{\phi}_{1}$ and $c\left(\varphi_{2}-\varphi_{1}\right)+b_{2}\left(\dot{\varphi}_{2}-\dot{\varphi}_{1}\right)$ are induced.

The kinetic energy $T$, the dissipation function $D$, the potential energy $V$, and the generalized forces $Q_{1}$ and $Q_{2}$ are:

$$
\begin{aligned}
& T=\frac{1}{2} m l^{2}\left(3 \dot{\phi}_{1}^{2}+2 \dot{\phi}_{1} \dot{\phi}_{2}+\dot{\phi}_{2}{ }^{2}\right) \\
& D=\frac{1}{2} b_{1} \dot{\phi}^{2}+\frac{1}{2} b_{2}\left(\dot{\phi}_{1}^{2}-2 \dot{\phi}_{1} \dot{\phi}_{2}+\dot{\phi}_{2}^{2}\right)
\end{aligned}
$$




$$
\begin{aligned}
& v=\frac{l}{2} c\left(2 \phi_{1}^{2}-2 \varphi_{1} m_{2}+q_{2}^{2}\right) \\
& Q_{1}=P l\left(q_{1}-\varphi_{2}\right) \\
& Q_{2}=0
\end{aligned}
$$

Lagrange's equations in the form

$$
\frac{d}{d t}\left(\frac{\partial T}{\partial \dot{\phi}_{i}}\right)+\frac{\partial D}{\partial \dot{\phi}_{1}}-\frac{\partial T}{\partial \phi_{i}}+\frac{\partial V}{\partial \varphi_{i}}=Q_{1} \quad(1=1,2)
$$

are employed to establish the linear equations of potion

$$
\begin{aligned}
& 3 m l^{2} \ddot{\phi}_{1}+\left(b_{1}+b_{2}\right) \dot{\phi}_{1}-(P l-2 c) \phi_{1}+m l^{2} \dot{\phi}_{2}-b_{2} \dot{\phi}_{2}+(\dot{l} l-c) \Phi_{2}=0 \\
& m l^{2} \ddot{\phi}_{1}-b_{2} \dot{\phi}_{1}-c \phi_{1}+m l^{2} \ddot{\phi}_{2}+b_{2} \dot{\varphi}_{2}+c \Phi_{2}=0
\end{aligned}
$$

which, upon stipulating solutions of the form

$$
\sigma_{i}=A_{i} e^{\omega t} \quad(1=1,2)
$$

yield the characteristic equation

$$
p_{0} \Omega^{4}+p_{1} \Omega^{3}+p_{2} \Omega^{2}+p_{3} q+p_{4}=0
$$

with the coefficients

$$
\begin{aligned}
& p_{0}=2 \\
& p_{1}=B_{1}+6 B_{2} \\
& p_{2}=7-2 F+B_{1} B_{2} \\
& p_{3}=B_{1}+B_{2} \\
& p_{4}=1
\end{aligned}
$$

and the dimensionless quantities 


$$
\begin{aligned}
& \Omega=\ell\left(\frac{\mathrm{m}}{\mathrm{c}}\right)^{\frac{1}{2}} \mathrm{w} \\
& \mathrm{B}_{1}=\frac{\mathrm{b}_{1}}{\ell(\mathrm{cm})^{\frac{1}{2}}} \quad(1=1,2) \\
& \mathrm{F}=\frac{\mathrm{Pl}}{\mathrm{c}}
\end{aligned}
$$

In the absence of damoing $\left(B_{1}=B_{2}=0\right)$, the characteristic equation is a biquadratic

$$
2 Q^{4}+(7-2 F) Q^{2}+1=0
$$

\section{Gritical Loads}

From the assumed form of the time-dependence for the coordinates $\varphi_{1}$ and on the basis of the kinatic stability criterion, it is evident that if all four roots of the characteristic equation are distinct, the necessary and sufficient conditions for stability are that the real roots and the real parts of the complex roots should be all negative or zero. In case of equal roots tine general solution of $\varphi_{i}$ will have terms which contain powers of $t$ as a factor. If the real parts of equal roots are negative, the system will be stable (vibration with decreasing amplitude), but if these real parts are zero or positive, stability will not exist (vibration with increasing amplitude).

Turning our attention first to the case of an infitially undamped system, the four roots of the biquadratic equation as a function of $P$ are

$$
Q_{1,2,3,4}=\frac{1}{2}[ \pm \sqrt{P-(7 / 2-\sqrt{2})} \pm \sqrt{F-(7 / 2+\sqrt{2})}]
$$

and are plotted in Fig. 2. The profections of the root exres on the 
real plane $(\operatorname{Im} \Omega=0)$, the Imapinary plane $($ Re $=0)$ and the complex plane $(F=0)$ are also shown in Fig. 2.

It is found that there will always be two roots with positive real part if $F>\frac{7}{2}-\sqrt{2}=2.086=F_{e}$. For $F=F_{\theta}$ there extst two pairs of equal roots whose real parts are all zero. Thus the system is unstable for $F \geq F_{e}$. For $F<F_{e}$ all roots are distinct ad pure imaginary and thus the system is stable. A further discussion of an initially undamped system is presented in [5].

We consider next a slightly damped system, assuming $B_{1}=B_{2}=0.01$. No simple expressions for the four roots of the quartic equation exist; the numerical results obtained are illustrated in Fig. 3 where a perspective view is supplemented by three projections on the same three planes as in Fig. 2. Two roots will have a positive real part for $F>1.464=F_{\mathrm{d}}$.

Stability can be investigated directly without determining the roots of the characteristic equation by applying the Routh-Hurwitz criteria [6], which require that all coefficienta $p_{j}(j=0, \ldots, 4)$ of the characteristic equation and the quantities

$$
\begin{aligned}
& s=p_{1} p_{2}-p_{0} p_{3} \\
& x=p_{1} p_{2} p_{3}-p_{0} p_{3}^{2}-p_{1}^{2} p_{4}
\end{aligned}
$$

be positive. For positive damping these stabdlity conditions are satisfled, provided

$$
p_{2}=2\left[-P+\frac{1}{2}\left(7+B_{1} B_{2}\right)\right]>0
$$


$\boldsymbol{t}$

6

$$
\begin{aligned}
& S=2\left(B_{1}+6 B_{2}\right)\left\{-F+\left[\frac{5\left(B_{2}+8 B_{2}\right)}{2\left(B_{1}+6 B_{2}\right)}+\frac{1}{2} B_{1} B_{2}\right]\right\}>0 \\
& x=2\left(B_{1}^{2}+7 B_{1} B_{2}+6 B_{2}^{2}\right)\left\{-F+\left[\frac{4 B_{1}^{2}+33 B_{1} B_{2}+4 B_{2}^{2}}{2\left(B_{1}^{2}+7 B_{1} B_{2}+6 B_{2}^{2}\right)}+\frac{1}{2} B_{1} B_{2}\right]\right\}>0
\end{aligned}
$$

For the system to be stable $F$ must satisfy the following three inequalities, where $\beta=B_{1} / B_{2}, 0 \leq \beta \leq \infty$

$$
\begin{aligned}
& F<\frac{7}{2}+\frac{1}{2} B_{1} B_{2} \\
& F<\frac{5(\beta+8)}{2(\beta+6)}+\frac{1}{2} B_{1} B_{2} \\
& F<\frac{4 B^{2}+33 B+h}{2\left(\beta^{2}+7 \beta+6\right)}+\frac{1}{2} B_{1} B_{2}
\end{aligned}
$$

Since

$$
\begin{aligned}
& \frac{5(\beta+8)}{2(\beta+6)}=\frac{5}{2}+\frac{5}{\beta+6} \leq \frac{10}{3}<\frac{7}{2} \\
& \frac{4 \beta^{2}+33 \beta+4}{2\left(\beta^{2}+7 \beta+6\right)}=\frac{5(\beta+8)}{2(\beta+6)}-\frac{\beta+3}{2(\beta+1)}<\frac{5(\beta+8)}{2(\beta+6)}<\frac{7}{2}
\end{aligned}
$$

for whatever $\beta$ in its range, it is evident that the critical load will be governed by the third inequality, 1.0.,

$$
\bar{F}_{d}=\frac{4 \beta^{2}+33 \beta+4}{2\left(\beta^{2}+7 \beta+6\right)}+\frac{1}{2} B_{1} B_{2}
$$

which depends on the ratio as well as the magnitudes of the damping coefficients.

For $B_{1} \ll 1$, as well as in the limit of vanishing damping, $\bar{F}_{d}$ becomes 


$$
F_{d}=\frac{4 \beta^{2}+33 \beta+1}{2\left(\beta^{2}+7 \beta+6\right)}
$$

which is highly dependent on $\beta$ and is in general smaller but never larger than $F_{e}$. The ratio of $F_{d}$ to $F_{0}$ versus $\beta$ is plotted in Fig. 4 . It is noted that when $\beta=4+5 \sqrt{2}=21.07, F_{d} / F_{0}$ reaches its maximur value 1. The destabilizing offect is thus eliminated in this particular case, similar to that found by Bolotin [4]. For $\beta=0, F_{d} / F_{0}$ reaches its minimuse value 0.16 ; 1.0 , the maximum destabillizing effect is about 846 in the present two-degreo-of-freedon ageten.

The Case of Vanishine Damping

The two disparate values of the critical load for no damping $\left(B_{1}=0\right)$ and vanishing damping $\left(B_{1} \rightarrow 0\right)$ justify a more detailed inrestigation of the limiting process as the damping coefficients approach zero.

Let us examine first the limiting process for the roots of the characteristic equation. It can be shown with the aid of the theory of equations [7] that if $B_{1} \ll 1$ and $F<4.914$ this equation will have four complex roots. Let these roots be

$$
\Omega=\left\{\begin{array}{l}
Y_{1} \neq i Y_{2} \\
\lambda_{1} \pm i \lambda_{2}
\end{array}\right.
$$

Then one can write [6], [7]

$$
2\left(r_{1}+\lambda_{1}\right)=-\frac{p_{1}}{p_{0}}
$$




$$
\Delta r_{1} \lambda_{1}\left[\left(r_{1}+\lambda_{1}\right)^{2}+\left(r_{2}+\lambda_{2}\right)^{2}\right]\left[\left(r_{1}+\lambda_{1}\right)^{2}+\left(r_{2}-\lambda_{2}\right)^{2}\right]=\frac{x}{p_{0}^{3}}
$$

where $P_{0}, P_{1}$ and $X$ are as defined earller. For vanishing damping

$$
\left\{\begin{array}{l}
r_{1}+\lambda_{1}=0 \\
r_{2} \lambda_{1}\left[\left(r_{1}+\lambda_{1}\right)^{2}+\left(r_{2}+\lambda_{2}\right)^{2}\right]\left[\left(r_{1}+\lambda_{1}\right)^{2}+\left(r_{2}-\lambda_{2}\right)^{2}\right]=0
\end{array}\right.
$$

Hence $r_{1}=-\lambda_{1}, r_{2}=\lambda_{2}$

or $\quad r_{1}=\lambda_{1}=0$

Thus

$$
\Omega=\left\{\begin{array}{l} 
\pm i r_{2} \\
\pm i \lambda_{2}
\end{array} \quad \text { or } \quad Q=\left\{\begin{array}{l}
r_{1} \pm i r_{2} \\
-r_{1} \pm i r_{2}
\end{array}\right.\right.
$$

and a substitution of these four roots into the characteristic equation will show that they are the same as in the case of no damping.

In the case of $F 24.914$, the four roots will be all real for small $B_{i}$. Let

$$
\Omega=\left\{\begin{array}{l}
u_{1} \neq u_{2} \\
v_{1} \neq v_{2}
\end{array}\right.
$$

In the limit of ranishing damping one can show similarly that either $u_{1}=v_{1}=0$ or $u_{1}=-v_{1}$ and $u_{2}=v_{2}$. For either alternative, subst1tution into the characteristic equation reveals that the roots are the same as in the case of no damping.

Thus the conclusion is roeched that whatever $F$ the roots of the characteristic equation for no inftial damping $\left(B_{1}=0\right)$ are identical to those of ranishing damping $\left(B_{1} \rightarrow 0\right)$. This 1 mplies that the motions 
of the system, for some given initial conditions, and whatever $F$, will be identical in the case of no damping $\left(B_{1}=0\right)$ and vanishing damping, $\left(\mathrm{B}_{1} \rightarrow 0\right)$.

We focus attention next on the loading $F$ in the two cases and before passing to the limit consider small damping $\left(B_{i} \ll 1\right)$. The positive real part of the roots of the characteristic equation in the range $\vec{F}_{\mathrm{e}}<\mathrm{F}<\mathrm{F}_{\mathrm{d}}$ for several small values of $B_{2}$ and, as an example, $B_{1}=0$ (i.e. $\beta=0$ ) have been calculated and the results are displayed in Fig. 5, where $F$ is plotted as a function of Res for 9 values of $B_{2}$. This Figure illustrates that for the larger values of $B_{2}, F_{d}$ represents the critical load because for $F>F_{d}$ some roots will have a non-vanishing positive real part. A small increase of the load above $F_{d}$ will result in a large increase of this real part. For small values of $B_{2}$, however, even though $F_{d}$ is still strictly speaking the critical load, its significance is lessened, because a small increase of the load above $F_{d}$ will not result any longer in a large increase of Res. Large increase of Re $\Omega$ will now be associated with small increase of a load which is slightly lower than $F_{e^{*}}$. For vanishing danping $R e \Omega=0$ for any $F<F_{e}$. We thus conclude that during the limiting process the significance of $F_{d}$ as a critical load is gradually transferred to $F_{e}$, and at the limit of vanishing damping $\left(B_{i} \rightarrow 0\right) F_{e}$ has to be considered as the critical load. It is apparent now that this conclusion could only be reached by considering the roots of the characteristic equation and not by merely applying the stability criteria of Routh-Hurwitz. Further, the reasons for the stability criteria jlelding different critical loads for no damping and for vanishing damping can bo bettor understood by having considerod small damping. 


\section{Degrce of Instability}

It was established in the proceding section that for vanishing dnmping $\left(B_{1} \rightarrow 0\right)$ the four roots of the characterlstio equation become identical to those of no damping $\left(B_{1}=0\right)$ while the stability criteria alone would in general yield disparate critical loads in these two cases.

To establish a further connection between the mathematically derived critical loads for no damping $\left(B_{i}=0\right)$ and vanishing damping $\left(B_{i} \rightarrow 0\right)$ it appears helpful to introduce into the discussion a concept which might be called "degree of instability" and which embodies a relaxation of the concept of instability as used when applying the kinetic stability criterion. According to this latter criterion a system is stable if a bounded

suitable disturbance results in a motion in the vicinity of the equilibriur configuration, e.g., the system is unstable if a disturbance leads to oscillations with increasing amplitude (flutter instability). For this type of loss of stability one can state that from a practical point of view it will certainly matter how fast the amplitudes increase.

For example, should a suitable initial disturbance be merely doubled in a time interval which is large as compared to, say, some reference period, while the duration of the system being subjected to a nonconservative force is by comparison relatively short, the system may be considered practically stable, while, mathematically of course, one would have to conclude that it is unstable.

In order to weaken the kinetic stability criterion, one could prescribe arbitrarfly the allowable increase of the disturbance and would then obtain for a given ralue of the load a critical tine, not unlike 
in the case of creep buckling. As an alternative, one could introduce another measure of the rate of amplitude 1ncrease. By analogy to decaying oscillations, where the logarithmic decrement serves the purpose of quantitatirely assessing the rate of decay, we can use the same quantity also as a measure of the rate of amplitude inorcase. Thus

$$
s=\log \frac{A_{n}}{A_{n+1}}
$$

where $A_{n}$ is the amplitude of the oscillation at a certain time $t$ and $A_{n+1}$ is the amplitude at $t+T$, where $T$ is the period. In the present problem, neglecting the terms of decaying magnitude in the general solution of $\varphi_{i}$, o will generally be time-independent for flutter motions, except when the characteristic equation has equal pure imaginary roots.

The kinetic stability criterion requires $8 \geq 0$ (1.e. $\left.A_{n} \geq A_{n+1}\right)$. A negative o could properly be called the logarithmic increment and in a real system it is conceivable that $\delta$ may attain a certain value ${ }_{c}{ }_{c}$ in a certain interval of time without the systen losing its stability in any practical sense.

For $\beta=B_{1} / B_{2}=1$ the critical load $F$ is displayed as a function of $B_{1}=B_{2}=B$ in Figs. 6 and 7. For however small but finite negative value of $b$, the critical load for vanishing damping $(B \rightarrow 0)$ will always be that for no damping $(B=0)$, namely $F_{\theta^{*}}$. However, the critical load for small damping $(B<1)$ may be smaller than $F_{\theta}$ but for finite 8 , however small, is always larger than $F_{d}$. For given o the value of (small) damping B which is associated with the minimu value of the critical load can be dotornined. 
For vanishing logarithmic increment $(b \rightarrow 0)$ the function $\vec{F}(B)$ approaches a limiting curve which will contain the point $F_{\mathrm{d}}$ on the ordinate. For $d=0$ the stability region 18 closed, 1.e., points on the curve $0=0$ in Fig. 7 are stable, including the point $F_{d}$ on the

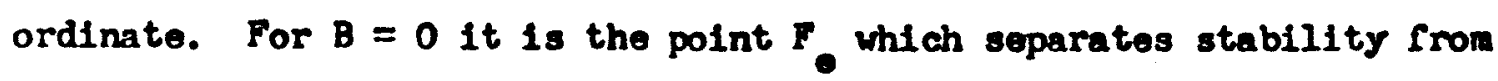
instability, but belongs itself to the instability rogion. This 11miting process provides thus additional inalght into the generation of the critical load $F_{d}$.

\section{Continuous Cantilever}

An attempt will be made now to interpret the results of the preceding sections, established with the aid of a simple two-degree-offreedom model, as applied to a continuous cantilever beam, which represents possibly a more reelistic system. This interoretation, however, is not without difficulty.

We shall assume that the internal damping of the contimuous cantilever can be represented by Voigt elements, 1.e., we use the Sezawa beam theory [8], and consider only the two lower modes of motion. The ordinary differential equation governing each mode $x_{1}$, of a cant1lever with no force at the free end, is of the form

$$
\ddot{x}_{1}+\frac{r w_{1}^{2}}{E} \dot{x}_{1}+w_{1}^{2} x_{1}=0
$$

where $Y$ is the damping coefficlent in the stress-strain relations and $E$ is Young's modulus. The ratio of the damping coefficiente of the first two nodes is thus 


$$
\bar{\beta}=\frac{w_{1}^{2}}{w_{2}^{2}}=\left(\frac{1.875}{4.694}\right)^{4}=0.0256
$$

This ratio for the continuous cantilever should now be compared with that of the cantilever model and for this purpose one should uncouple the two equations governing the model. It is known [9.], however, thnt whenever a dissipation energy is accounted for, in addition to kinetic and potential energies, such uncoupling can, in general, not be effected and this is the difficulty alluded to above. In the system under consideration uncoupling becomes possible in the special case given by $\beta=1$ (1.e., $b_{1}=b_{2}=b$ ) because in this case the dissipation function becores proportional to the potential energs.

The transformation

$$
\begin{aligned}
& p_{1}=\frac{1}{\sqrt{2}} y_{1} \\
& p_{2}=-\frac{1}{\sqrt{2}} y_{1}+y_{2}
\end{aligned}
$$

with

$$
\begin{aligned}
& y_{1}=\xi_{1} \cos \theta-\xi_{2} \sin \theta \\
& y_{2}=\xi_{1} \sin \theta+\xi_{2} \cos \theta
\end{aligned}
$$

leads to the uncoupled equations

$$
\begin{aligned}
& \ddot{\xi}_{1}+\frac{b}{4 m l^{2}}(7-\sqrt{41}) \dot{\epsilon}_{1}+\frac{c}{4 m l^{2}}(7-\sqrt{41}) \xi_{1}=0 \\
& \ddot{\xi}_{2}+\frac{b}{4 m l^{2}}(7+\sqrt{41}) \dot{\zeta}_{2}+\frac{c}{4 m l^{2}}(7+\sqrt{41}) \varepsilon_{2}=0
\end{aligned}
$$

In this representation the ratio of the damping coefficients is given by

$$
\overline{\bar{\beta}}=\frac{7-\sqrt{47}}{7+\sqrt{41}}=0.0446
$$


The $\bar{\beta}$ and $\bar{\beta}$ are relatively close and one can conclude that in the original coordinates $\varphi_{i}$ the ratio of the damping coefficients $\beta$ has to be taken in the vicinity of unity to correspond to the continuous cantilever.

Further, for many structural materials the fraction of critical damping $\varepsilon_{i}=\frac{r w_{i}}{2 E}$ is lonown to be of the order of $10^{-3}$.

$$
\begin{aligned}
& \text { Since (with } \beta=1 \text { ) } \\
& 2 E_{1} b_{1}=\frac{b}{4 m l^{2}}(7-\sqrt{41})
\end{aligned}
$$

and

$$
w_{1}=\frac{\sqrt{c}}{2 L} \sqrt{m} \sqrt{(7-\sqrt{41})}
$$

the fraction of critical damping in the first mode will be

$$
\varepsilon_{1}=\mathrm{B} \sqrt{7-\sqrt{41}}=0.775 \mathrm{~B}
$$

Similarly, for the second mode it will be

$$
\varepsilon_{2}=3.661 \mathrm{~B}
$$

Thus $B$ is of the same order of magnitude as $\varepsilon_{1}$, 1.e. $10^{-3}$, and damping will have indeed a destabilizing affect, as seen from Fig. 6.

\section{Concludine Remarks}

An examination of the roots of the characteristic equation and the introduction of the concept of degree of instability make it possible to estabifsh a generic relationship between the critical loads for no damping and for semall and vanishing damping. Routh-Hurwitz criteria alone proved to be insufficient to determine the critical load for 
vanishing damping, which is the same as for no damping. It is small damping, rather than vanishing damping, which is responsibio for the destabilizing effect. The strong dependence of the critical load on the ratio of the damping coefficients, however, leaves a requirement for further investigation, which should include other damping mechanisn, effects of nonlineartty and different types of nonconservative forces.

\section{REPERENCES}

1. H. Ziegler, Die Stabilitätskriterien der Elastomechanik," Incenieur-Archiv, vol. 20, 2952, pp. 49-56.

2. H. Ziegler, ULinear Mastic Stability," Zeitschrift für anrewandte Nathematik und Phrsik, vol. 4, 1953, pp. 89-121, 168-185.

3. H. Zieglet, "On the Concedt of Elastic Stability," Advances in Applied Mechanjes, vol. 4, edited by K. I. Dryden and T. von Karman, Acadenic Press, Inc., New York, N. Y., 1956, pp. 351-403.

4. V. V. Bolotin, Nonconservative Problems of the Theory of Elastic Stability, Moscow, 1961; English translation published by Pergamon Press, Inc., New York, N. Y., 1963.

5. G. Hermann and R. W. Bungay, "On the Stability of Hlastic Systems Subjected to Nonconservative Forces," Jounnal of Applied Mechanics, vol. 31,1964 , pp. $435-440$.

6. E. J. Routh, Advanced Dymamics of a System of Ripid Bodies, Dover Publications, New York, N. Y., 1955.

7. W. S. Burnside and A. W. Panton, The Theory of Equztions, 10th. ed., Dublin, Hodges, Figgls, \& Co., 1935.

8. R. D. Mindlin, F. W. Stubner, and H. L. Cooper, "Response of Damped Elastic Systens to Transient Disturbances," Proceedinas of the Society for Experimental Stress Analysts, vol. 5, No. 2, 1948, pp. 69-87.

9. J. W. S. Rayleigh, The Theory of Sound, Dover Publications, New York, N. Y., 2945 . 


\section{CAPTIONS OF FIGURES}

1. Two-degree-of-freedom model

2. Orthographic projections and the perspective of the root curves of the characteristic equation with no damping

3. Ortinographic projections and the perspective of the root curves of the characteristic equation with damping

4. Critical load versus ratio of damping coefficients for $B_{i} \ll I$

5. Significance of the critical load $F_{d}$ as $B_{2}$ increases

6. Critical load for various degrees of instability versus small demping coefficients

7. Critical load for various degrees of instability versus large damping coeficients. 
Herimann and Jong

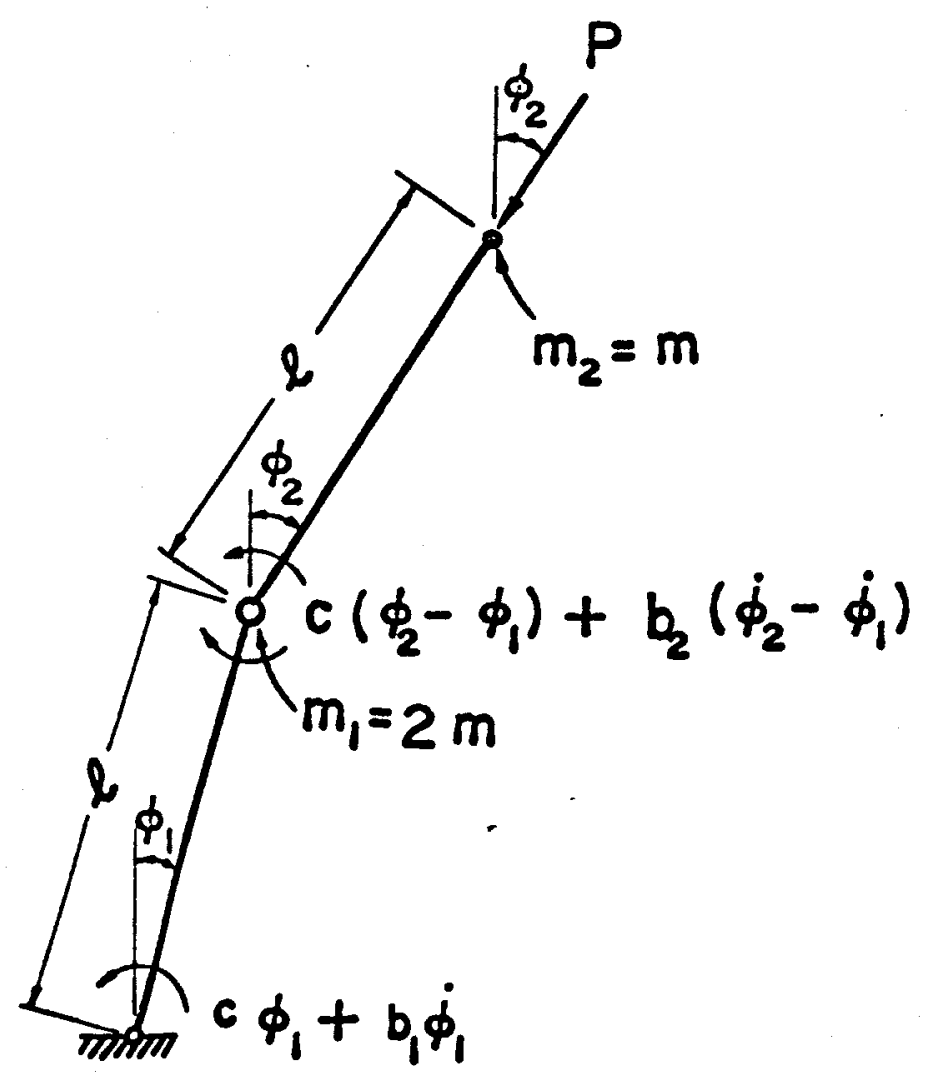

Fig. I 

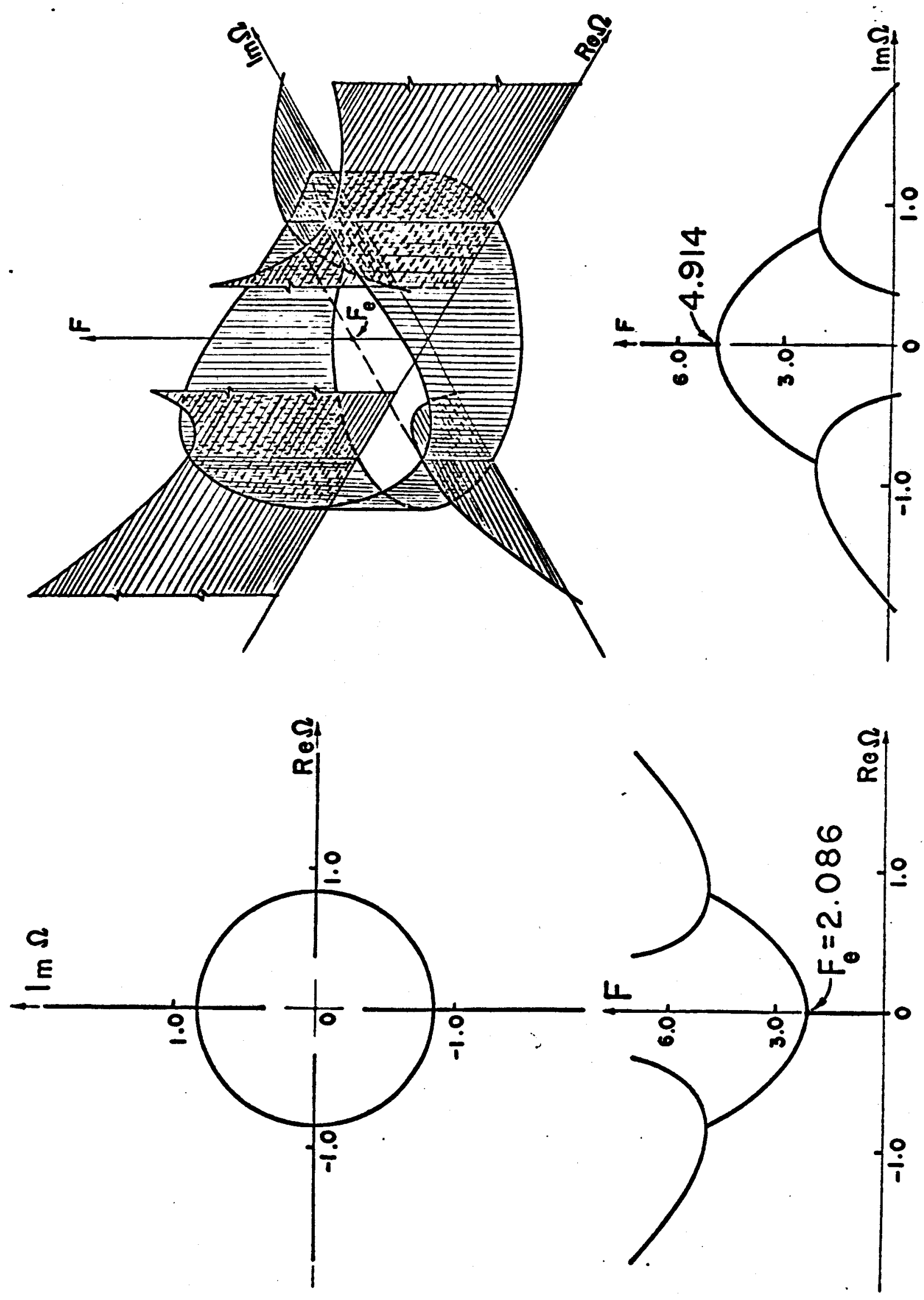


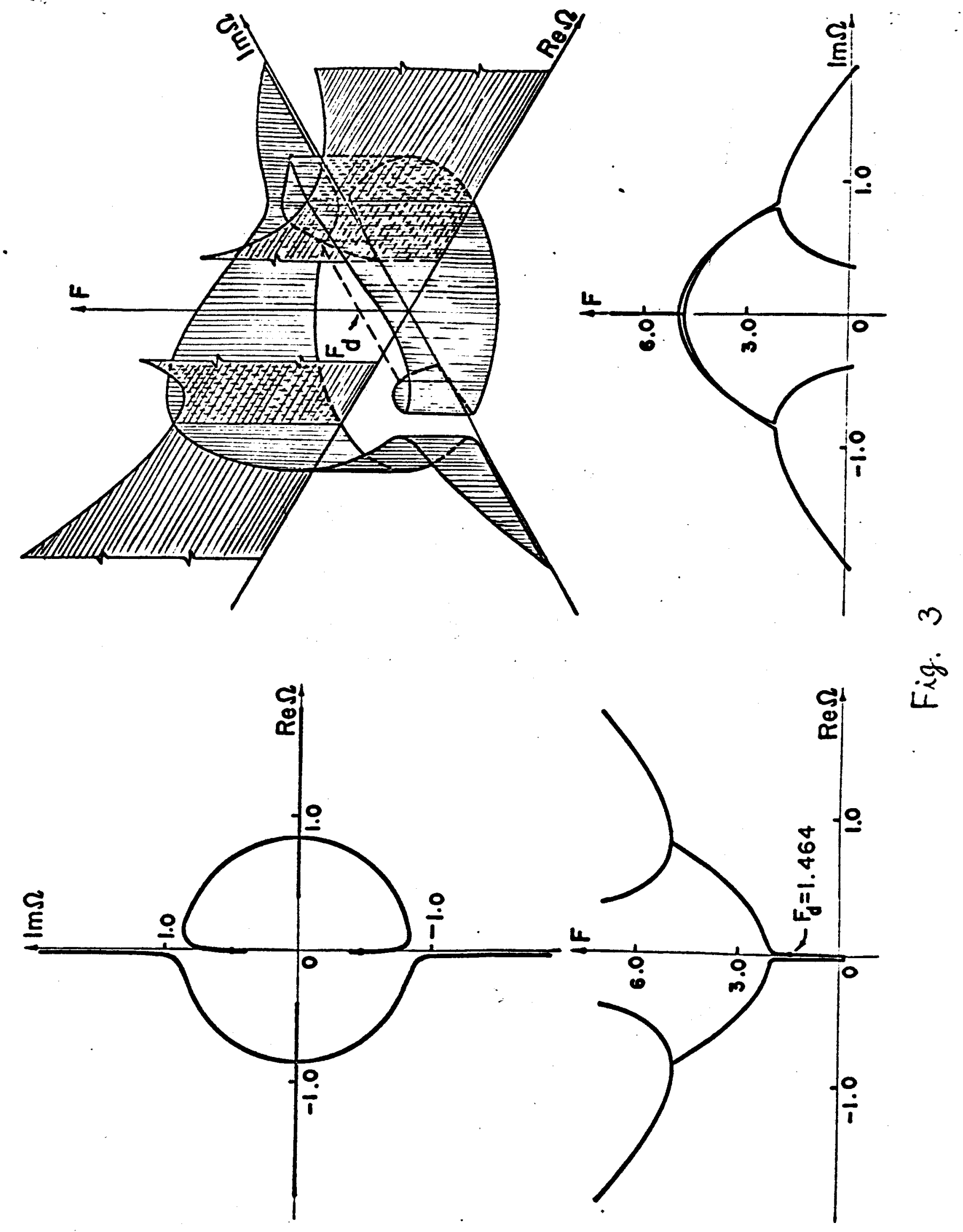


$\infty$

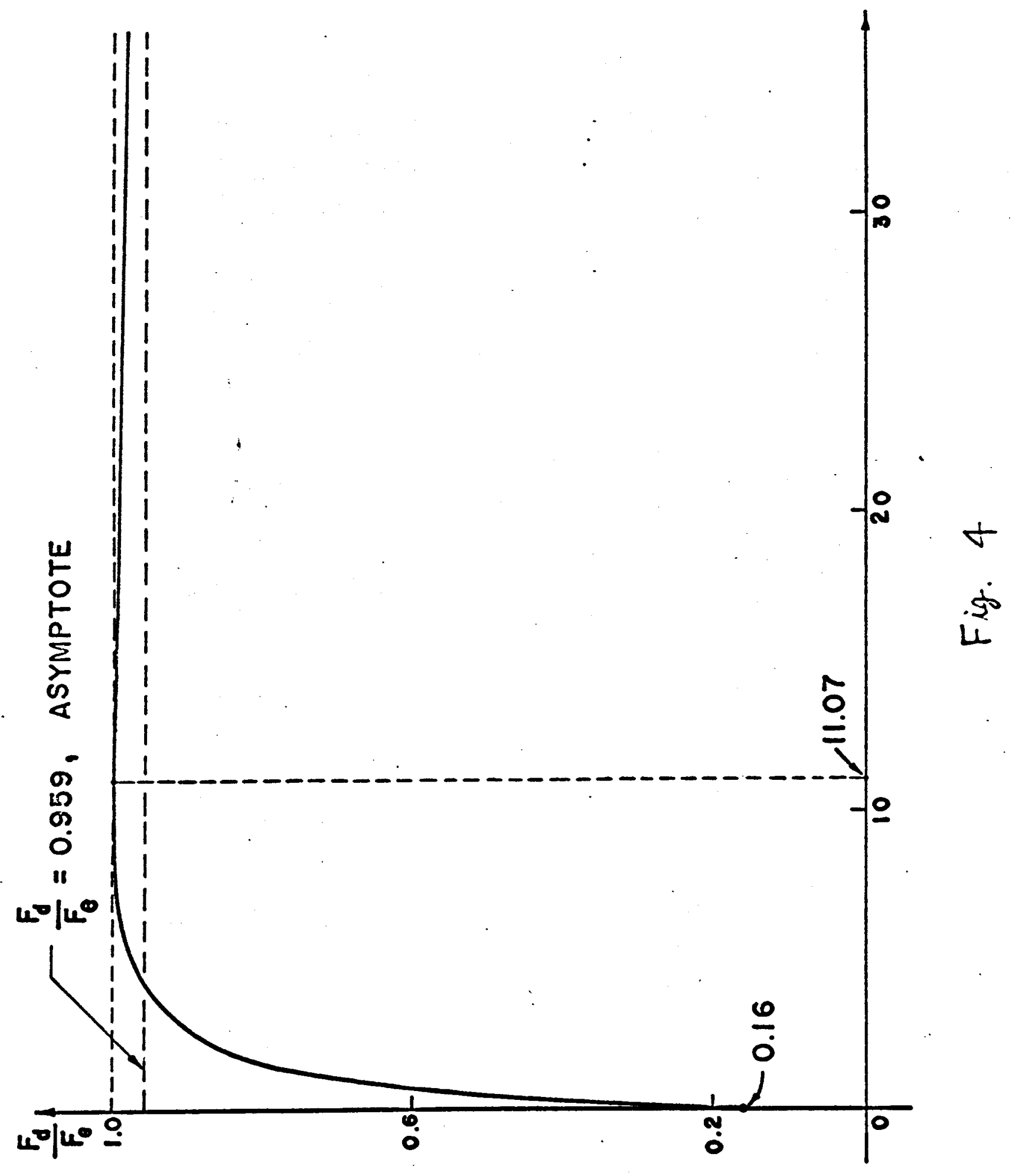




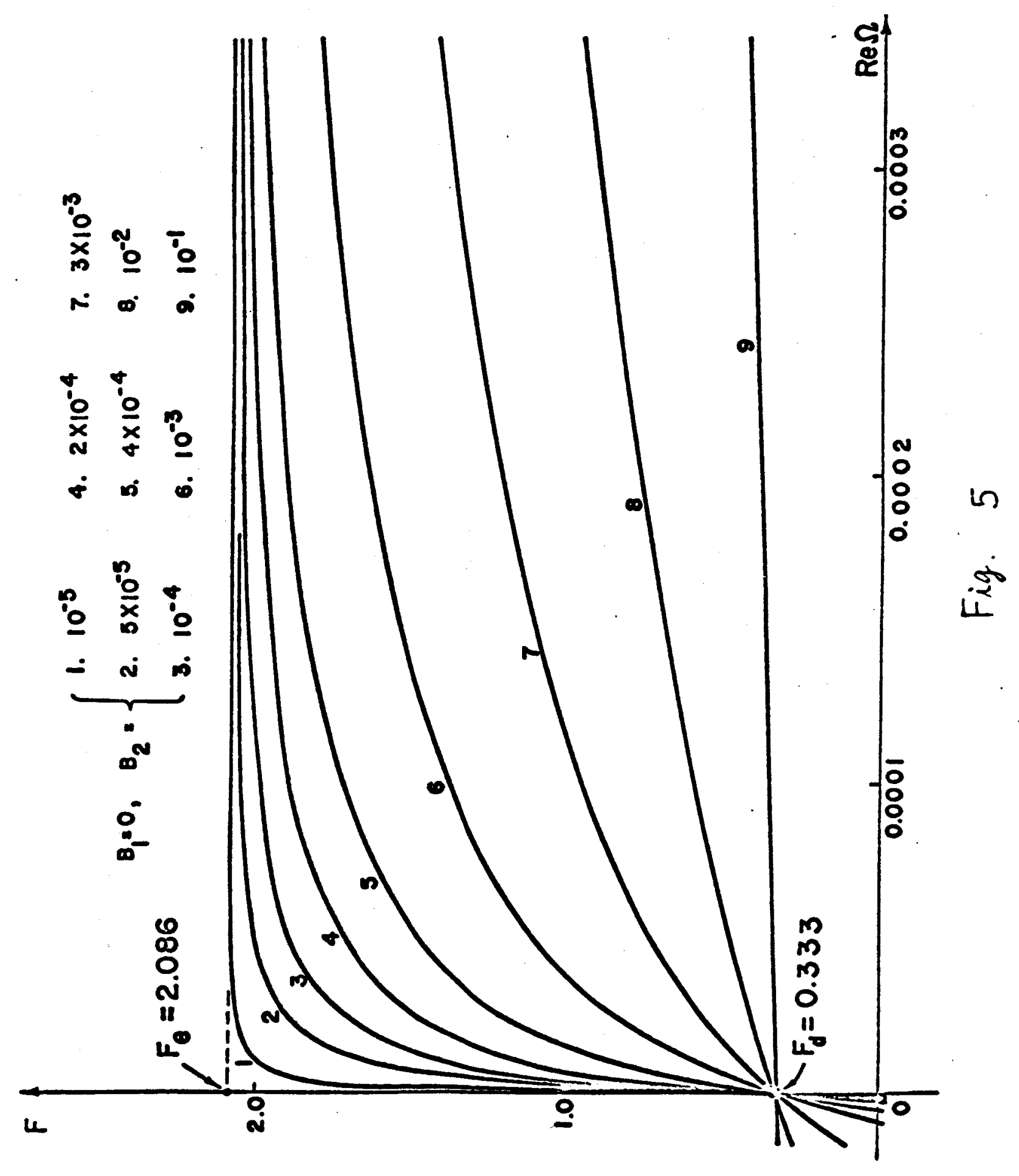


$\infty$

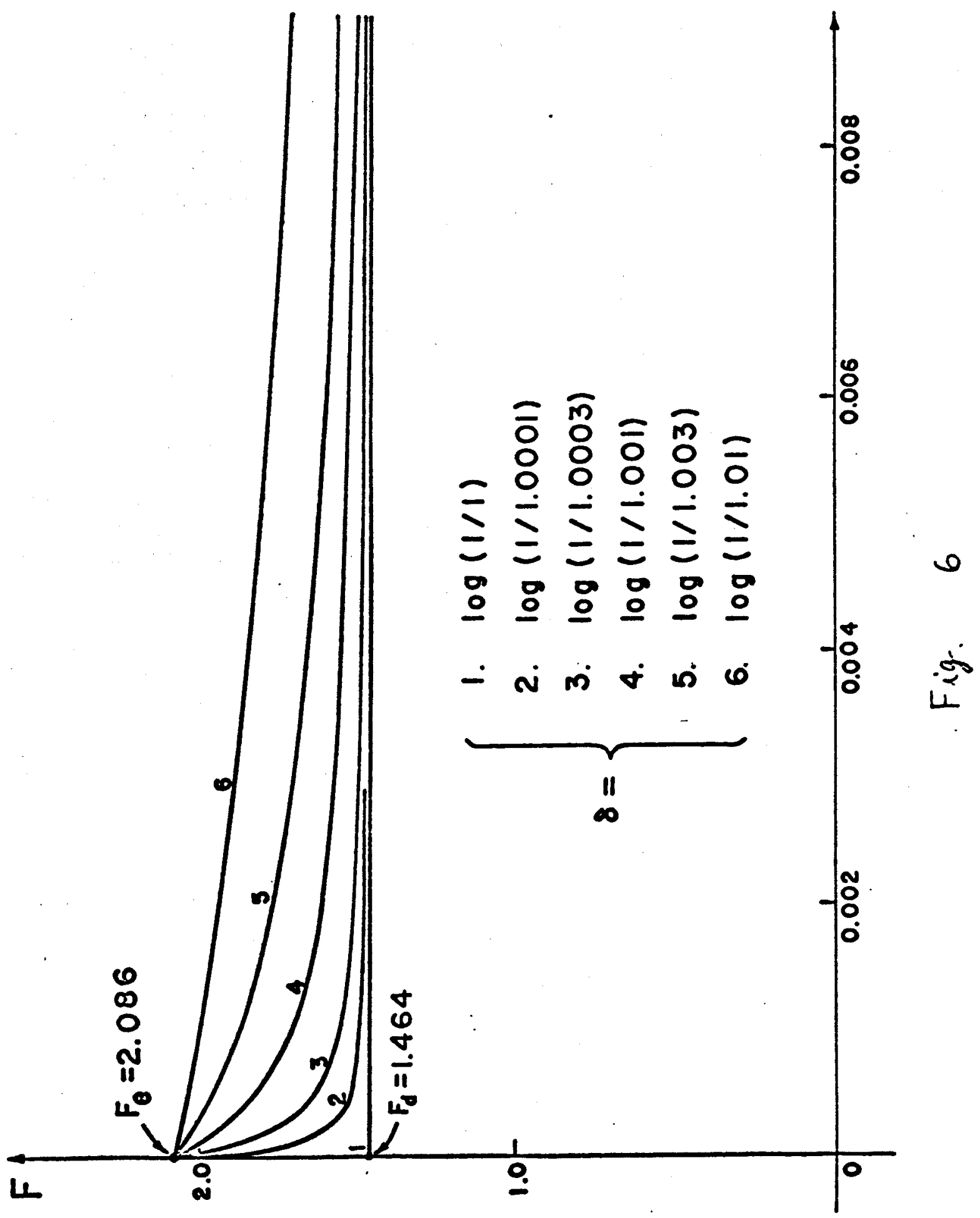

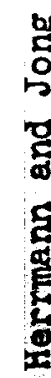




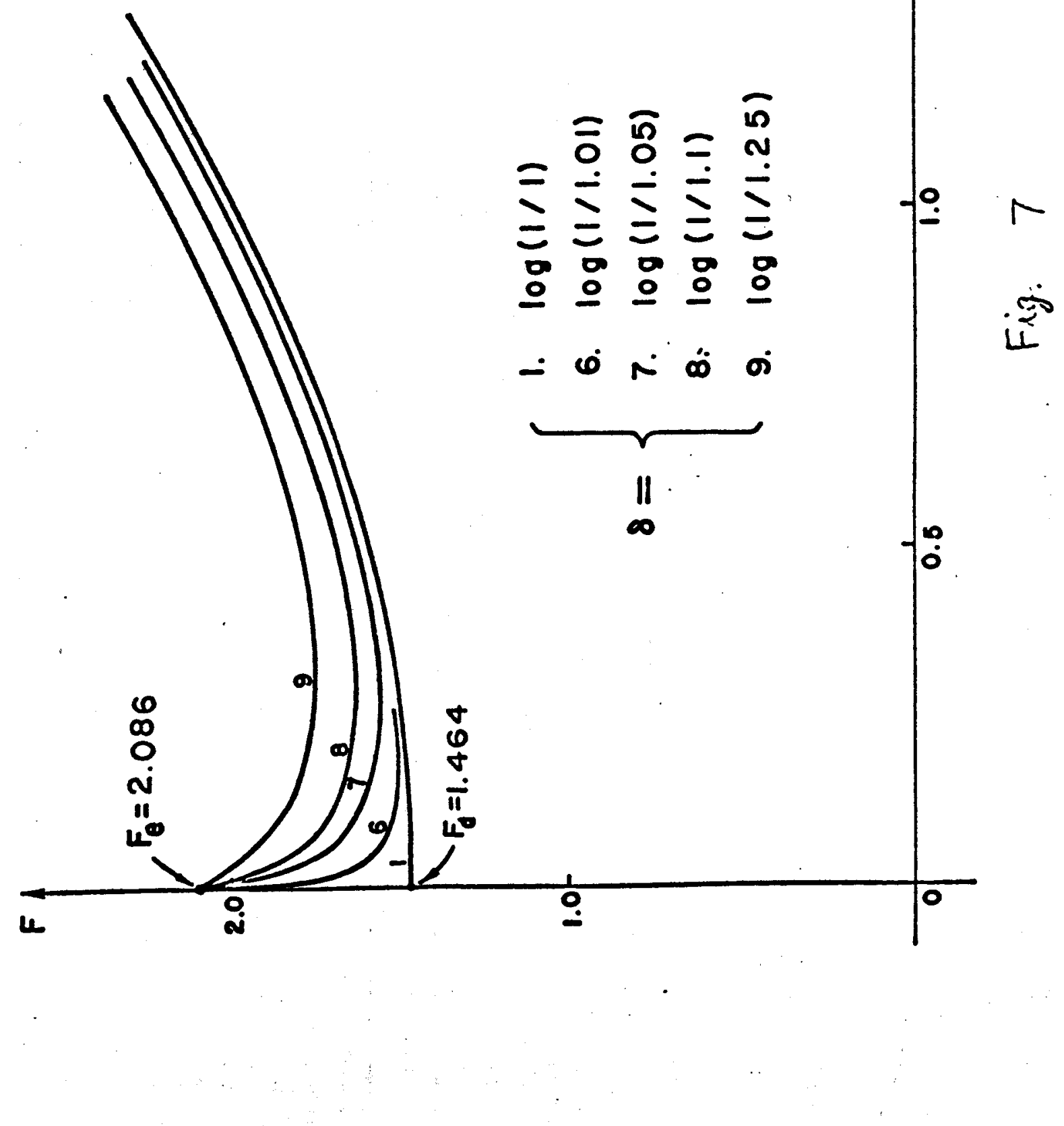




\section{SUMMARY}

The destabilizing effect of linear viscous damping in a nonconservative elastic system is imrestigated by studying the roots of the characteristic equation in addition to the stability criteria and by introducing the concept of degree of instability. A generic relationship between critical loadings for no damping and for slight damping as well as ranishing damping is established. It is found that while the presence of small damping may have a destabilizing effect, proper interpretation of the liniting process of rantshing damping leads to the same critical load as for no damping. 


\section{Introduction}

It has been discovered by Ziegler [1] a little more than a decade ago, that internal damping may have a destabilizing offect in nonoonservative elastic system. He considered a double pendulum with viscoelastic hinges as a model of an elastic bar with internal damping and let a tangential force act at the free end. The critical loading obtained in complete absence of damping was found to be considerably higher than by including damping at the outset of the analysis and then letting the damping coefficients approach zero (vanishing damping) in the expression for the critical force.

This rather surprising and paradoxical finding was ascribed in later studies by Ziegler [2], [3] to the possibility that internal damping is inadequately represented by linear damping forces which are linear combinations of the generalized velocities and that the hysteresis effect should be taken into account.

The destabilizing effect of damping was further elaborated upon by Bolotin [4] who considered a general two-degree-of-freedom system not related to ans particular mechanical model and who found additionally that the destabilizing effect in the presence of slight and vanishing damping is highly dependent on the relative magnitude of damping coefficients in the two degrees freedom.

It is the aim of the present investigation to make an attempt at supplying some additional insight into the destabilizing affects of linear relocity-dependent damping in nonconservative systems, without raising the question here as to the suitabdifty of this damping mechanism 
2

for a realistic system. For this purpose the system discussed by Ziegler is reconsidered, and not only the stability conditions are investigated but also the roots of the characteristic equations themselves. Plots of these roots for various ranges of loading illustrate graphically how the paradoxical effects of vanishing damping are generated. Further, the results of the mathematical stability investigations are interpreted in physical terms by introducing the concept of degree of instability.

These concepts permit to carry out a gradual transition from the case of small damping to the case of vanishing damping and relate them to the case of no damping. Finally, some remarks are made with regard to possible behavior of an elastic bax with distributed parameters.

The Model

We consider a double pendulum, Fig. 1, composed of two rigid weightless bars of equal length $l$, which carry concentrated masses $m_{1}=2 m$, $m_{2}=m$. The generalized coordinates $\varphi_{1}, \varphi_{2}$ are taken to be small. A load $P$ applied at the free end is assumed to be acting at an angle $\Phi_{2}$ (follower force). At the hinges the restoring moments $c \varphi_{1}+b_{1} \dot{\varphi}_{1}$ and $c\left(\varphi_{2}-\varphi_{1}\right)+b_{2}\left(\dot{\varphi}_{2}-\dot{\varphi}_{1}\right)$ are induced.

The kinetic energy $T$, the dissipation function $D$, the potential energy $v$, and the generalized forces $Q_{1}$ and $Q_{2}$ ares

$$
\begin{aligned}
& T=\frac{1}{2} m l^{2}\left(3 \dot{\phi}_{1}^{2}+2 \dot{\phi}_{1} \dot{\phi}_{2}+\dot{\phi}_{2}^{2}\right) \\
& D=\frac{1}{2} b_{1} \dot{\phi}^{2}+\frac{1}{2} b_{2}\left(\dot{\phi}_{1}^{2}-2 \dot{\phi}_{1} \dot{\phi}_{2}+\dot{\phi}_{2}^{2}\right)
\end{aligned}
$$




$$
\begin{aligned}
& v=\frac{l}{2} c\left(2 \Phi_{1}^{2}-2 \phi_{1} \Phi_{2}+\varphi_{2}^{2}\right) \\
& Q_{1}=P l\left(\varphi_{2}-\varphi_{2}\right) \\
& Q_{2}=0
\end{aligned}
$$

Lagrange's equations in the form

$$
\frac{\partial}{d t}\left(\frac{\partial T}{\partial \dot{\phi}_{1}}\right)+\frac{\partial D}{\partial \dot{\phi}_{1}}-\frac{\partial T}{\partial \phi_{1}}+\frac{\partial V}{\partial \phi_{i}}=Q_{1} \quad(1=1,2)
$$

are employed to establish the linear equations of motion

$$
\begin{aligned}
& 3 m l^{2} \ddot{\phi}_{1}+\left(b_{1}+b_{2}\right) \dot{\phi}_{1}-(P l-2 c) \phi_{1}+m l^{2} \ddot{\phi}_{2}-b_{2} \dot{q}_{2}+(P l-c) \phi_{2}=0
\end{aligned}
$$

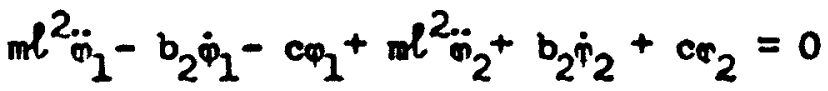

which, upon stipulating solutions of the form

$$
\sigma_{i}=A_{i} e^{\omega t} \quad(i=1,2)
$$

yield the characteristic equation

$$
p_{0} d^{4}+p_{1} Q^{3}+p_{2} \Omega^{2}+p_{3} Q+p_{4}=0
$$

with the coefficients

$$
\begin{aligned}
& p_{0}=2 \\
& D_{1}=B_{1}+6 B_{2} \\
& p_{2}=7-2 F+B_{1} B_{2} \\
& p_{3}=B_{1}+B_{2} \\
& P_{4}=1
\end{aligned}
$$

and the dimensionless quantities 


$$
\begin{aligned}
& Q=\ell\left(\frac{m_{c}}{c}\right)^{\frac{2}{2}} w \\
& B_{1}=\frac{b_{1}}{\ell(c m)^{\frac{1}{2}}} \quad(1=1,2) \\
& F=\frac{P l}{c}
\end{aligned}
$$

In the absence of dombing $\left(B_{1}=B_{2}=0\right)$, the characteristic equation is a biquadratic

$$
2 \Omega^{4}+(7-2 F) Q^{2}+1=0
$$

\section{Critical Loads}

From the assumed form of the time-dependence for the coordinates $\varphi_{i}$ and on the basis of the kinetic stability criterion, it is evident that if all four roots of the characteristic equation are distinct, the necessary and sufficient conditions for stability are that the real roots and the real parts of the complex roots should be all negative or zero. In case of equal roots tine general solution of $\varphi_{i}$ will have terms which contain powers of $t$ as a factor. If the real parts of equal roots are negative, the system will be stable (vibration with decreasing amplitude), but if these real parts are zero or positive, stability will not oxist (ribration with increasing amplitude).

Turning our attention first to the case of an initially undamped system, the four roots of the biquadratio equation as a function of $P$ are

$$
\Omega_{1,2,3,4}=\frac{1}{2}[ \pm \sqrt{7-(7 / 2-\sqrt{2})} \pm \sqrt{7-(7 / 2+\sqrt{2})}]
$$

and ars plotted in Pig. 2. The projectioñ of the root curres on the 
real plane $(\operatorname{Im} \Omega=0)$, the imapinary plane $(\operatorname{Re} \Omega=0)$ and the complex plane $(F=0)$ are also shown in Fig. 2 .

It is found that there will always be two roots with positive real part if $F>\frac{7}{2}-\sqrt{2}=2.086=F_{e}$. For $F=F_{e}$ there exist two pairs of equal roots whose real parts are all zero. Thus the system is unstable for $F \geq F_{e}$. For $F<F_{e}$ all roots are distinct ad pure imaginary and thus the system is stable. A further discussion of an initially undamped system is presented in [5].

We consider next a slightly damped system, assuming $B_{1}=B_{2}=0.01$. No simple expressions for the four roots of the quartic equation exist; the numerical results obtained are illustrated in Fig. 3 where a perspective view is supplemented by three projections on the same three planes as in Fig. 2. Two roots will have a positive real part for $F>1.464=F_{\text {d }}$.

Stability can be investigated directly without determining the roots of the characteristic equation by applying the Routh-Hurwitz criteria [6], which require that all coefficients $p_{j}(j=0, \ldots, 4)$ of the characteristic equation and the quantities

$$
\begin{aligned}
& s=p_{1} p_{2}-p_{0} p_{3} \\
& x=p_{1} p_{2} p_{3}-p_{0} p_{3}^{2}-p_{1}{ }^{2} p_{4}
\end{aligned}
$$

be positive. For positive damping these stability conditions are satisfied, provided

$$
p_{2}=2\left[-F+\frac{I}{2}\left(7+B_{1} B_{2}\right)\right]>0
$$


6

$$
\begin{aligned}
& S=2\left(B_{1}+6 B_{2}\right)\left\{-F+\left[\frac{5\left(B_{1}+B B_{2}\right)}{2\left(B_{1}+6 B_{2}\right)}+\frac{1}{2} B_{1} B_{2}\right]\right\}>0 \\
& x=2\left(B_{1}^{2}+7 B_{1} B_{2}+6 B_{2}^{2}\right)\left\{-F+\left[\frac{4 B_{1}^{2}+33 B_{1} B_{2}+4 B_{2}^{2}}{2\left(B_{1}^{2}+7 B_{1} B_{2}+6 B_{2}^{2}\right)}+\frac{1}{2} B_{1} B_{2}\right]\right\}>0
\end{aligned}
$$

For the system to be stable $F$ mast satisfy the following throe inequalities, where $\beta=B_{1} / B_{2}, 0 \leq \beta \leq \infty$

$$
\begin{aligned}
& F<\frac{7}{2}+\frac{1}{2} B_{1} B_{2} \\
& F<\frac{5(\beta+8)}{2(\beta+6)}+\frac{1}{2} B_{1} B_{2} \\
& F<\frac{4 B^{2}+33 \beta+L}{2\left(\beta^{2}+7 \beta+6\right)}+\frac{1}{2} B_{1} B_{2}
\end{aligned}
$$

Since

$$
\begin{aligned}
& \frac{5(\beta+8)}{2(\beta+6)}=\frac{5}{2}+\frac{5}{\beta+6} \leq \frac{10}{3}<\frac{7}{2} \\
& \frac{4 \beta^{2}+33 \beta+4}{2\left(\beta^{2}+7 \beta+6\right)}=\frac{5(3+8)}{2(\beta+6)}-\frac{\beta+3}{2(\beta+1)}<\frac{5(\beta+8)}{2(\beta+6)}<\frac{7}{2}
\end{aligned}
$$

for whatever $\beta$ in its range, it is evident that the critical load will be governed by the third inequality, 1.e.,

$$
\bar{F}_{d}=\frac{4 \beta^{2}+33 \beta+4}{2\left(\beta^{2}+7 \beta+6\right)}+\frac{1}{2} B_{1} B_{2}
$$

which depends on the ratio as wall as the magnitudes of the damping coefficients.

For $B_{1} \ll 1$, as well as in the limit of vanishing damping, $\bar{F}_{d}$ becomes 


$$
F_{d}=\frac{4 \beta^{2}+33 \beta+1}{2\left(\beta^{2}+7 \beta+6\right)}
$$

which is highly dependent on $\beta$ and is in general smaller but never larger than $F_{e}$. The ratio of $F_{d}$ to $F_{\theta}$ versus $\beta$ is plotted in $F i g .4$. It is noted that when $\beta=4+5 \sqrt{2}=11.07, F_{d} / F_{0}$ reaches its maximum value 1. The destabilizing of fect is thus eliminated in this particular case, similar to that found by Bolotin [4]. For $\beta=0, F_{d} / F_{0}$ reaches its minimun value 0.16; 1.e., the maximum destabilizing effect is about $84 \%$ in the present two-degree-of-freedom systen.

\section{The Case of Vanishing Damping}

The two disparate values of the critical load for no damping $\left(B_{1}=0\right)$ and vanishing damping $\left(B_{1} \rightarrow 0\right)$ justify a more detailed investigation of the limiting process as the damping coefficients approach zero.

Let us examine first the limdting process for the roots of the characteristic equation. It can be shown with the ald of the theory of equations [7] that if $B_{1} \ll 1$ and $F<4.914$ this equation will have four corplex roots. Let these roots be

$$
\Omega=\left\{\begin{array}{l}
r_{1} \pm 1 r_{2} \\
\lambda_{1} \pm 1 \lambda_{2}
\end{array}\right.
$$

Then one can urite $[6],[7]$

$$
2\left(r_{1}+\lambda_{1}\right)=-\frac{p_{1}}{p_{0}}
$$




$$
\left\langle r_{1} \lambda_{1}\left[\left(r_{1}+\lambda_{1}\right)^{2}+\left(r_{2}+\lambda_{2}\right)^{2}\right]\left[\left(r_{1}+\lambda_{1}\right)^{2}+\left(r_{2}-\lambda_{2}\right)^{2}\right]=\frac{x}{p_{0}^{3}}\right.
$$

where $p_{0}, p_{1}$ and $X$ are as defined earlier. For vanishing damping

$$
\left\{\begin{array}{l}
r_{1}+\lambda_{1}=0 \\
r_{1} \lambda_{1}\left[\left(r_{1}+\lambda_{1}\right)^{2}+\left(r_{2}+\lambda_{2}\right)^{2}\right]\left[\left(r_{1}+\lambda_{2}\right)^{2}+\left(r_{2}-\lambda_{2}\right)^{2}\right]=0
\end{array}\right.
$$

Hence $r_{1}=-\lambda_{1}, r_{2}=\lambda_{2}$

or $\quad r_{1}=\lambda_{1}=0$

Thus

$$
Q=\left\{\begin{array}{l} 
\pm i r_{2} \\
\pm i \lambda_{2}
\end{array} \quad \text { or } \quad Q=\left\{\begin{array}{l}
r_{1} \pm i r_{2} \\
-r_{1} \pm i r_{2}
\end{array}\right.\right.
$$

and a substitution of these four roots into the characteristic equation vill show that they are the same as in the case of no damping. In the case of $F \geq 4.914$, the four roots will be all real for $\operatorname{small~} B_{i}$. Let

$$
a=\left\{\begin{array}{l}
u_{1} \neq u_{2} \\
v_{1} \neq v_{2}
\end{array}\right.
$$

In the limit of vanishing damping one can show similarly that either $u_{1}=v_{1}=0$ or $u_{1}=-v_{1}$ and $u_{2}=v_{2}$. For either alternative, substitution into the characteristic equation reveals that the roots are the same as in the case of no damping.

Thus the conclusion is reached that whatever $F$ the roots of the characteristic equation for no initial damping $\left(B_{1}=0\right)$ are identical to those of vaniahting dauping $\left(B_{1} \rightarrow 0\right)$. This implies that the motions 
of the system, for some given initial conditions, and whatever $F$, will be identical in the case of no damping $\left(B_{1}=0\right)$ and vanishing damping $\left(B_{i} \rightarrow 0\right)$.

We focus attention next on the loading $F$ in the two cases and before passing to the limit consider small damping $\left(B_{i} \ll 1\right)$. The positive real part of the roots of the characteristic equation in the range $F_{e}<F<F_{d}$ for several small values of $B_{2}$ and, as an example, $B_{1}=0$ (i.e. $\beta=0$ ) have been calculated and the results are displayed in Fig. 5, where $F$ is plotted as a function of Res for 9 values of $B_{2}$. This Figure illustrates that for the larger values of $B_{2}, F_{d}$ represents the critical load because for $F>F_{d}$ some roots will have a non-vanishing positive real part. A small increase of the load above $F_{d}$ will result in a large increase of this real part. For small values of $B_{2}$, however, even though $F_{d}$ is still strictly speaking the critical load, its significance is lessened, because a small increase of the load above $F_{d}$ will not result any longer in a large increase of Res. Large increase of Re $\Omega$ will now be associated with small increase of a load which is slightly lower than $F_{e}$. For vanishing damping ReQ $=0$ for any $F<F_{e^{*}}$. We thus conclude that during the limiting process the significance of $F_{d}$ as a critical load is gradually transferred to $F_{e}$, and at the limit of vanishing damping $\left(B_{i} \rightarrow 0\right) F_{e}$ has to be considered as the critical load. It is apparent now that this conclusion could only be reached by considering the roots of the characteristic equation and not by merely applying the stability criteria of Routh-Hurwitz. Further, the reasons for the stabillty criteria fielding different critical loads for no damping and for vanishing damping can be better understood by having considered small damping. 


\section{Degree of Instability}

It was established in the preceding section that for vanishing damping $\left(B_{1} \rightarrow 0\right)$ the four roots of the charaoteristio equation becomo identical to those of no damping $\left(B_{1}=0\right)$ while the stability criteria alone would in general yield disparate critical loads in these two cases.

To establish a further connection between the mathematically derived critical loads for no damping $\left(B_{1}=0\right)$ and vanishing damping $\left(B_{i} \rightarrow 0\right)$ it appears helpful to introduce into the discussion a concept which might be called "degree of instability" and which embodies a relaxation of the concept of instability as used when applying the kinetic stability criterion. According to this latter criterion a system is stable if a bounded

suitable disturbance results in $a_{A}$ motion in the vicinity of the equilibrium configuration, e.g., the system is unstable if a disturbance leads to oscillations with increasing amplitude (flutter instability). For this type of loss of stability one can state that from a practical point of view it will certainly matter how fast the amplitudes increase.

For example, should a suitable initial disturbance be merely doubled in a time interval which is large as compared to, say, some reference period, while the duration of the system being subjected to a nonconservative force is by comparison relatively short, the system may be considered practically stable, while, mathematically of course, one would have to conclude that it is unstable.

In order to weaken the kinetic stability criterion, one could prescribe arbitrarily the ellowable increase of the disturbance and would then obtain for a giren ralue of the load a critical tine, not unlike 
in the case of creep buckling. As an alternative, one could introduce another measure of the rate of amplitude 1ncrease. By analogy to decaying oscillations, where the logarithmic decrement serves the purpose of quantitatively assessing the rate of decar, we can use the same quantity also as a measure of the rate of amplitude inorease. Thus

$$
s=\log \frac{A_{n}}{A_{n+1}}
$$

where $A_{n}$ is the amplitude of the oscillation at a certain time $t$ and $A_{n+1}$ is the amplitude at $t+T$, where $T$ is the period. In the present problem, neglecting the terms of decaying magnitude in the general solution of $\varphi_{i}$, o will generally be time-independent for flutter motions, except when the characteristic equation has equal pure imaginary roots.

The kinetic stability criterion requires o $\geq 0$ (1.e. $A_{n} \geq A_{n+1}$ ). A negative o could properly be called the logarithmic incroment and in a real system it is conceivable that $\delta$ may attain a certain value $\delta_{c}$ in a certain interval of time without the system losing its stability in any practical sense.

For $\beta=B_{1} / B_{2}=1$ the critical load $F$ is displayed as a function of $B_{1}=B_{2}=B$ in Figs. 6 and 7. For however small but finite negative value of 8 , the critical load for vanishing damping $(B \rightarrow 0)$ will always be that for no damping $(B=0)$, namely $F_{\theta^{-}}$However, the critical load for small damping $(B<1)$ may be smaller than $F_{\theta}$ but for finite $b$, however small, is always larger than $F_{d}$. For given o the value of (small) damping $B$ which is associated with the minim value of the critical load can be doternined. 
For vanishing logarithmic increment $(\delta \rightarrow 0)$ the function $\vec{F}(B)$ approacies a limiting curve which will contain the point $F_{d}$ on the ordinate. For $\delta=0$ the stability region is closed, $1 . \theta_{.}$, points on the curve $\delta=0$ in Fig. 7 are stable, including the point $F_{d}$ on the ordinate. For $B=0$ it 18 the point $F_{\text {a }}$ which separates stability from instability, but belongs itself to the instability region. This Iimiting process provides thus additional insight into the generation of the critical load $F_{d}$.

Continuous Cantilever

An atteropt will be made now to interpret the results of the preceding sections, established with the aid of a simple two-degree-offreedom modẹl, as applied to a continuous cantilever beam, which represents possibly a more realistic system. This interpretation, however, is not without difficulty.

We shall assume that the internal damping of the contimous cantilever can be represented by Voigt elements, 1.e., we use the Sezawa beam theory [8], and consider only the two lower modes of motion. The ordinary differential equation governing each mode $x_{1}$, of a cantilever wth no force at the free end, is of the form

$$
\ddot{x}_{1}+\frac{r w_{1}^{2}}{E} \dot{x}_{1}+w_{1}^{2} x_{1}=0
$$

where $r$ is the damping coefficient in the stress-strain relations and E is Young's modulus. The ratio of the damping coefficients of the firat two nodes is thus 


$$
\bar{\beta}=\frac{w_{1}^{2}}{w_{2}^{2}}=\left(\frac{1.875}{4.694}\right)^{4}=0.0256
$$

This ratio for the continuous cantilever should now be compared with that of the cantilever model and for this purpose one should uncouple the two equations governing the model. It is known [9], however, that whenever a dissipation energy is accounted for, in addition to kinetic and potential energies, such uncoupling can, in general, not be effected and this is the difficulty alluded to above. In the system under consideration uncoupling becomes possible in the special case given by $\beta=1\left(1.0 ., b_{1}=b_{2}=b\right)$ because in this case the dispation function becores proportional to the potential energy.

The transformation

$$
\begin{aligned}
& \varphi_{1}=\frac{1}{\sqrt{2}} y_{1} \\
& \varphi_{2}=-\frac{1}{\sqrt{2}} y_{1}+y_{2}
\end{aligned}
$$

with

$$
\begin{aligned}
& y_{1}=\xi_{1} \cos \theta-\zeta_{2} \sin \theta \\
& y_{2}=\xi_{1} \sin \theta+\xi_{2} \cos \theta
\end{aligned}
$$

leads to the uncoupled equations

$$
\begin{aligned}
& \ddot{\xi}_{1}+\frac{b}{4 m l^{2}}(7-\sqrt{41}) \dot{\zeta}_{1}+\frac{c}{4 m l^{2}}(7-\sqrt{41}) \xi_{1}=0 \\
& \ddot{\xi}_{2}+\frac{b}{4 m l^{2}}(7+\sqrt{41}) \dot{\xi}_{2}+\frac{c}{4 m l^{2}}(7+\sqrt{41}) \xi_{2}=0
\end{aligned}
$$

In this representation the ratio of the damping coefficients is given by

$$
\bar{\beta}=\frac{7-\sqrt{41}}{7+\sqrt{41}}=0.0446
$$


The $\bar{B}$ and $\bar{\beta}$ are relatively close and one can conclude that in the original coordinates $\varphi_{i}$ the ratio of the damping coefficients $\beta$ has to be taken in the vicinity of unity to correspond to the continuous cantilever.

Further, for many structural materials the fraction of critical damping $\varepsilon_{i}=\frac{r w_{i}}{2 E}$ is lonown to be of the order of $10^{-3}$.

$$
\text { Since (with } \beta=1 \text { ) }
$$

$$
2 \varepsilon_{1} w_{1}=\frac{b}{4 m b^{2}}(7-\sqrt{41})
$$

and

$$
\omega_{1}=\frac{\sqrt{c}}{2 l \sqrt{m}} \sqrt{(7-\sqrt{41})}
$$

the fraction of critical damping in the first mode will be

$$
\varepsilon_{1}=B \sqrt{7-\sqrt{41}}=0.775 B
$$

Similarly, for the second mode it will be

$$
\varepsilon_{2}=3.661 \mathrm{~B}
$$

Thus $B$ is of the same order of magnitude as $\varepsilon_{i}, 1 . e .10^{-3}$, and damping will have indeed a destabilizing effect, as seen from Fig. 6.

\section{Concludine Remarks}

An examination of the roots of the characteristic equation and the introduction of the concept of degree of instability make it possible to establish a generic relationship between the critical loads for no damping and for small and vanishing damping. Routh-Hurwitz criteria alone proved to be insufficient to determine the critical load for 
vanishing damping, which is the same as for no damping. It is small damping, rather than vanishing damping, which is responsiblo for the destabilizing effect. The strong dependence of the critical load on the ratio of the damping coefficients, howerer, leaves a requirement for further investigation, which should include other damping mechanism, effects of nonlinearity and different types of nonconservative forces.

\section{REFERONCES}

1. H. Ziegler, "Die Stabjlitätskoriterien der Elastomechanik," Incenieur-Archiv, vol. 20, 1952, pp. 49-56.

2. H. Ziegler, "Linear Mastic Stability," Zeitschrift für anrewandto Mathematik und Phrsik, vol. 4, 1953, pp. 89-121, 168-185.

3. H. Ziegler, "On the Concedt of Flastic Stability," Advances in Applied Mechantes, vol. 4, edited by H. L. Dryden and T. von Karman, Academic Press, Inc., New York, N. Y., 1956, pp. 351-403.

4. V. V. Bolotin, Nonconservative Problems of the Theory of Elastic Stability, Moscow, 1961; English translation published by Pergamon Press, Inc., New York, N. Y., 1963.

5. G. Herrmann and R. W. Bungay, "On the Stability of Elastic Systems Subjected to Nonconservative Forces," Joumal of Applied Mechanics, vol. $31,1964, \mathrm{pp} .435-440$.

6. E. J. Routh, Aivanced Dynamics of a System of Rigid Bodies, Dover Publications, New York, N. Y., 1955.

7. W. S. Burnside and A. W. Panton, The Theory of Equations, 10th. ed., Dublin, Hodges, Figgis, \& Co., 1935.

8. R. D. Mindlin, F. W. Stubner, and H. L. Cooper, "Response of Damped Elastic Systems to Transient Disturbances," Proceodings of the Society for Bcoerimental Stress Analysis, vol. 5, No. 2, 1948, pp. 69-87.

9. J. W. S. Rayleigh, The Theory of Sound, Dover Publications, New York, N. Y., 2945. 


\section{GAPTIONS OF FIGURES}

1. Two-degree-of-freedom model

2. Orthographic projections and the perspective of the root curves of tine characteristic equation with no damping

3. Ortinographic projections and the perspective of the root curves of the characteristic equation with damping

4. Critical load versus ratio of damping coefficients for $B_{i} \ll I$

5. Significance of the critical load $F_{\mathrm{d}}$ as $B_{2}$ increases

6. Critical load for various degrees of instability versus small damping coofficients

7. Critical load for various degrees of instability versus large damping coefficients. 
Herimann and Jong

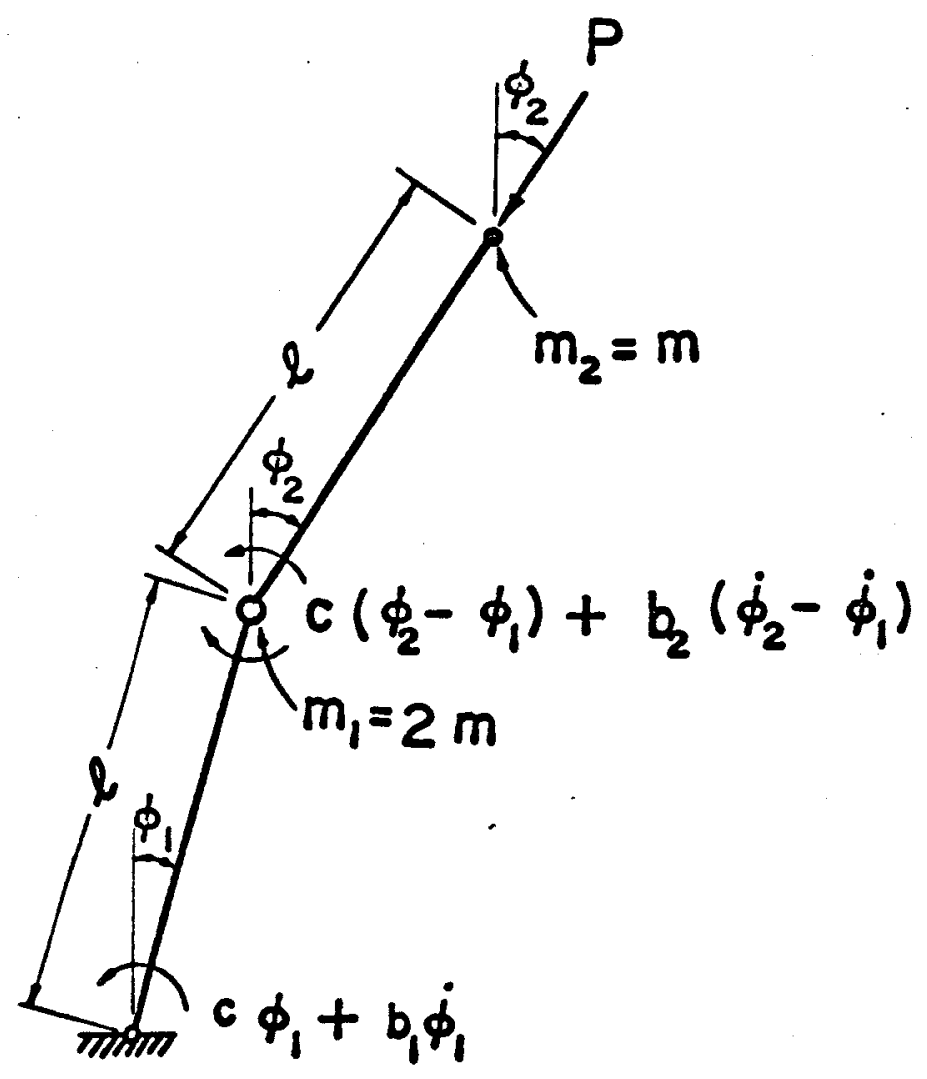

Fig. I 


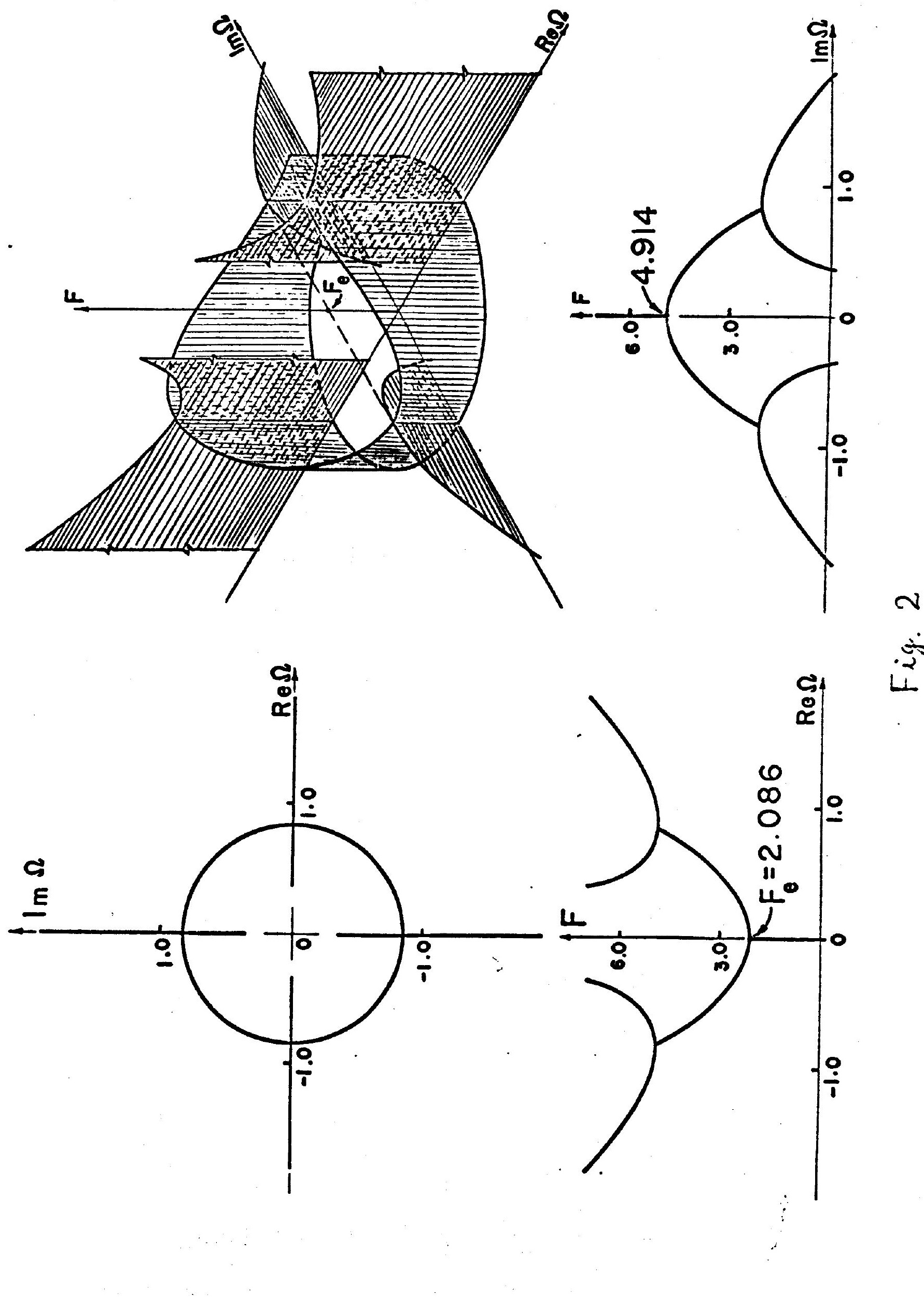




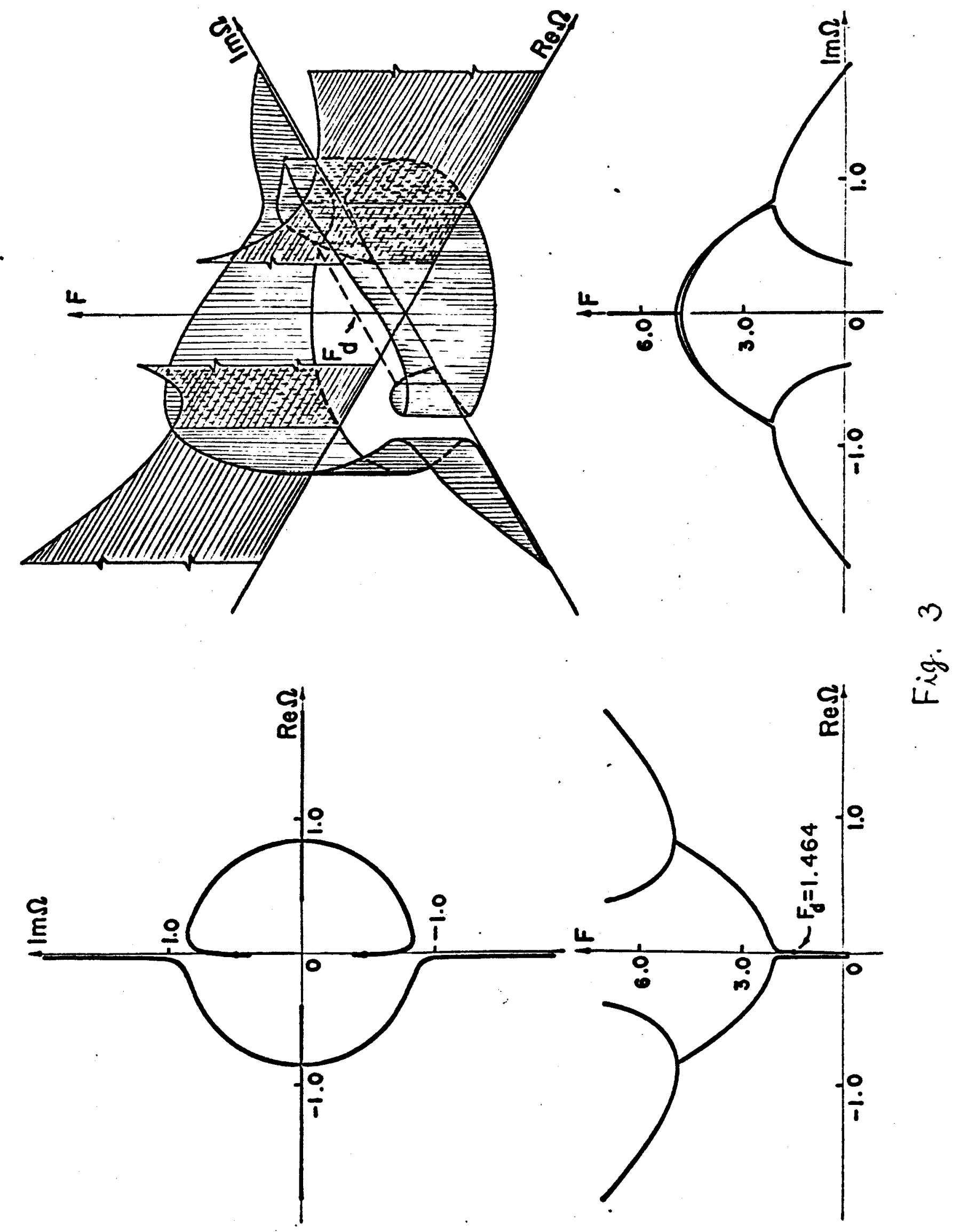




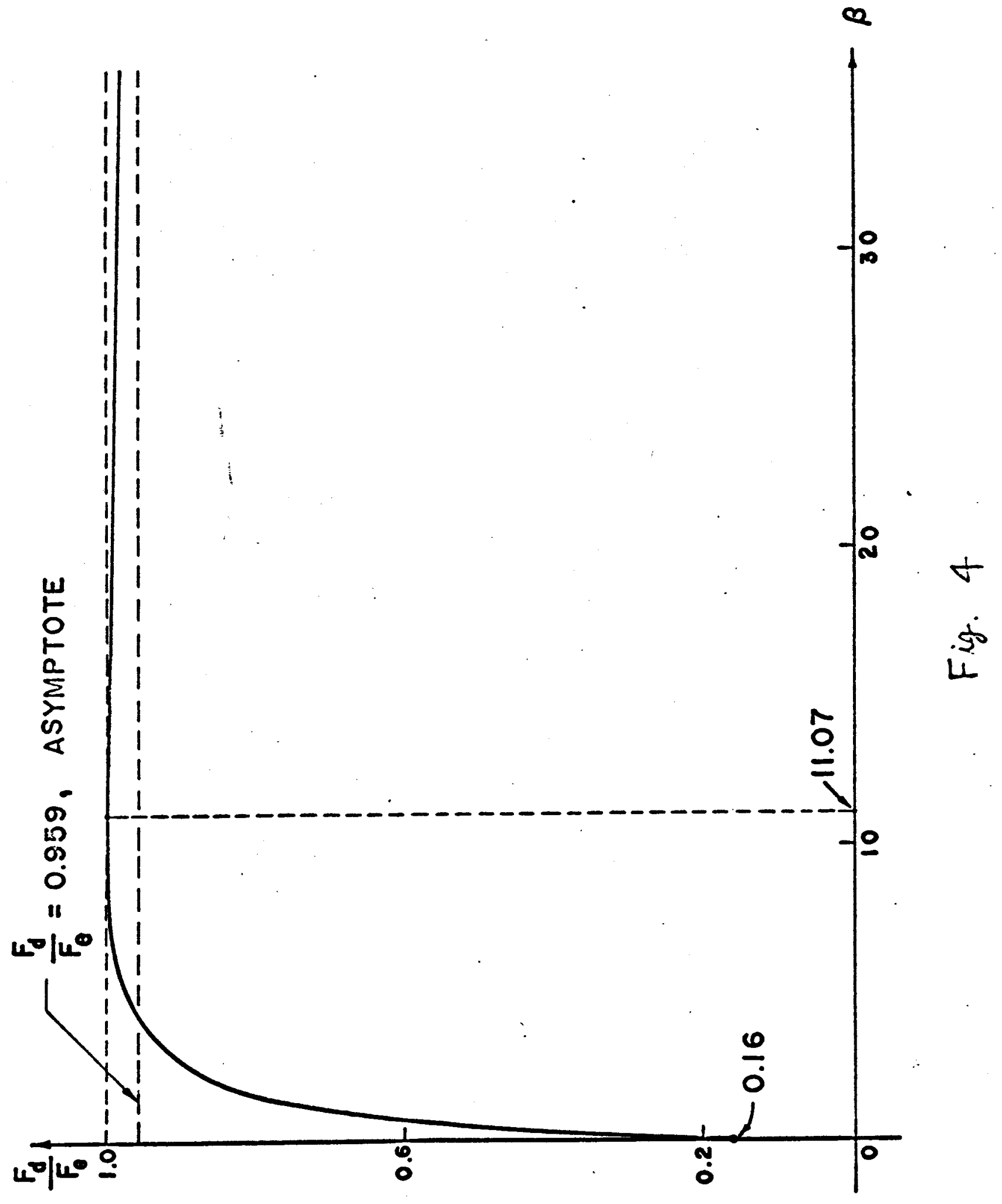





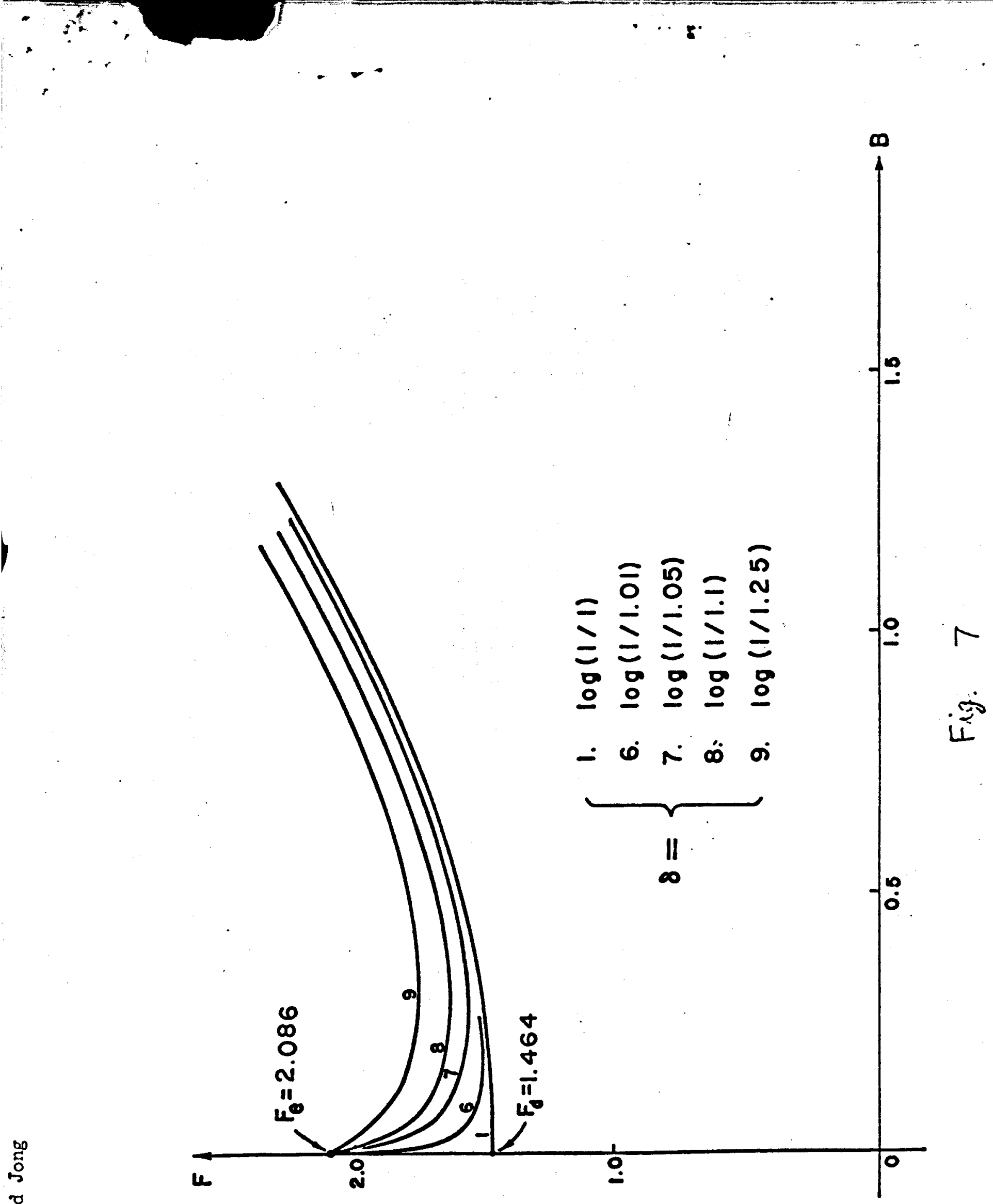